\title{
Realization of Intelligent Traffic Control Management System Based on Internet of Things RFID Technology
}

\author{
Yi Wang ${ }^{1,}$, Haoyuan $\mathrm{Ou}^{1}$ and Jianming Zhang ${ }^{1}$ \\ ${ }^{1}$ Department of Information Engineer, Guangdong Polytechnic, Foshan, China \\ awangyifsfz@163.com
}

Keywords: Intelligent transportation system; RFID; Internet of things; Control management system; Traffic signal lamp

\begin{abstract}
Intelligent transportation system (ITS) is an advanced RFID technology, cloud computing, mobile communication, automatic control, computer network technology and other organic applying to the whole traffic management system and set up a real-time, accurate, efficient transportation integrated management and control system. RFID is a non-contact automatic ID recognition technology, through the radio frequency signal to automatically identify the object of the ID number, fast, real-time, accurate processing of information. The paper presents realization of intelligent traffic control management system based on Internet of things RFID Technology. Finally, through the simulation of intelligent traffic light control system, the simulation results show that the system has good working performance.
\end{abstract}

\section{Introduction}

With nearly two years of networking technology in the rapid development of domestic, intelligent transportation field was given a more scientific and technological content, in technology and management philosophy is also caused by the revolutionary change [1]. ITS: Intelligent transport systems. This international term was formally recognized in projects as the "intelligent vehicle road system (IVHS)" (Vehicle Highway System Intelligent). With the intelligent traffic has become increasingly urgent, the modernization of transport industry increasingly onerous task to determine the road traffic intelligent research results applied to expand the development direction of railway, waterway, and aviation field, from the angle of the comprehensive transportation system to study the intelligent traffic network problems, which gradually formed a establish a new concept of intelligent transportation system.

Intelligent transportation is an integrated system, which includes the following aspects: first, the vehicle control system. System for assisting a driver is to drive a car or an alternative to a driver; Two, traffic monitoring system [2]. The system is similar to the airport's aviation controller; it will be in the road, the vehicle and the driver to establish a fast communication link.

Intelligent transportation system (ITS) is an advanced RFID technology, GPS technology, cloud technology, mobile communication technology, automatic control technology, computer network technology and other organic applying to the whole traffic management system and set up a real-time, accurate, efficient transportation integrated management and control system. It consists of several subsystem composition (such as free flow detection system, toll collection system, bus rapid transit system, signal control system, information release system, etc.) through the system and integrates Road, vehicles, pedestrians organic combined with together, to strengthen the links between the three, timely and accurate and efficient supervision of the traffic situation. In this paper, we put forward realization of intelligent traffic control management system based on Internet of things RFID Technology.

\section{Study on Application of Internet of Things RFID}

RFID is a non-contact automatic ID recognition technology, through the radio frequency signal to automatically identify the object of the ID number, fast, real-time, accurate acquisition, accurate 
processing of information. RFID tags have waterproof, antimagnetic, high temperature resistance, reading distance, storage information freely change, large storage capacity can tag data encryption, identification of moving objects can be, can be fast and convenient operation, so the RFID technology application in various fields and working environment.

Reader to launch a specific frequency of radio energy to Transponder, to drive the Transponder circuit will be sent inside the Code ID, at this time Reader will receive this Code ID. Transponder is special because free battery, contact free, free credit card, so are not afraid of dirty and wafer password for the world only, can not be copied, high safety and long service life [3]. RFID application is very wide, at present, the typical application of animal chips, car chip anti-theft devices, access control, parking control, production line automation, material management, etc...

The internal structure of RFID tag chip mainly includes four parts, which are RF front end, analog front end, digital baseband processing unit and EEPROM storage unit. Reader, also known as reader, reader, RFID (interrogator), is to read / write operation of the device, mainly including RF module and digital signal processing unit two parts, as is shown by equation (1).

$$
\phi_{M, i}(t)=\sum_{m=1}^{2^{L}} u_{m}^{L} \phi_{L, m}(t)+\sum_{j=L}^{M-1} \sum_{m=1}^{2^{j}} w_{m}^{j} \psi_{j, m}(t)
$$

Things are generally recognized as divided into three levels, the bottom is the perception of perception and front-end to collect data layer, the middle is the network layer data transmission and processing, the top is use through the analysis and processing of sensory data, for users provide various application services to the application layer.

Internet of things is all the items through the wireless radio frequency identification RFID and other information sensing devices connected with the Internet, to achieve intelligent network. With the passage of time and the development of technology, the connotation of the Internet of things has changed a great change.

RFID car tag, sensor network node tags; get event user login event, card record events, etc.. The formula used in the method is as follows [4].

$$
\mu_{s_{0}}=E_{k}\left\{s_{0}(k)\right\}=\sum_{k \in \mathcal{R}_{k}} p(k) s_{0}(\kappa)
$$

RFID system is generally composed of 3 layers of tag, reader and antenna. The tag layer is composed of a chip and a coupling element, and each tag has a unique electronic code, which is attached to the identification object. The electronic data in the prescribed format is kept in an electronic tag, and then the electronic tag is attached to the surface of the object [5]. The reader layer is a device for reading and writing tag information, which can read and write data in the electronic label without contact. Usually read and write device connected with the computer, read the information transmitted through the network to the computer for the next step. The antenna is used to transmit radio frequency signals between the tag layer and the reader layer.

In the Internet of things, the reader will be the same as the communication, control and Computing (communication, control, Computing) function of the $\mathrm{C} 3$ core equipment. Antenna (antenna) is the RFID tag and reader to achieve radio frequency signal space communication and the establishment of wireless communication devices.

The basic working principle of RFID technology is not complicated: label into the field after receiving an interpretation of a radio frequency signal, by induction current of energy to send out stored in the chip product information; the reader will read information and decoding, sent to the central information system on the data processing [6].

Network connection and business closely: the traditional Internet, the security of network layer and business layer is independent of each other, and in the Internet of things network layer and service layer has a close relationship, is tightly bound, which produced the transmission of information security and 
privacy problems in Internet of things, and privacy and security has also become a constraint network for the further development of the important reasons.

\section{Design and Analysis of Intelligent Traffic Signal Lamp and Control Management System}

Intelligent transportation system (ITS) is applied in urban traffic is mainly reflected in microscopic traffic information collection, traffic control and guidance, by increasing the effective use and management of traffic information to improve the efficiency of traffic system, mainly by the information acquisition input strategy, control system, and an output execution, between each subsystem data transmission and communication subsystems.

The architecture of traffic signal control system is described as follows: signal control center equipment mainly includes central control server, regional control server, communication server, database server, and client and so on. Communication part mainly includes optical and communication network, signal control point optical machine and equipment are used to connect, the communication interface RJ45. Intersection part of the equipment mainly includes signal machine, detector, signal according to the vehicle detector detected traffic information (including traffic) real-time adjustment of intersection control (signal period and green ratio), the realization of intersection of the orderly control scheme, as is shown by equation (3) [7].

$$
\frac{R^{n k} u^{(0)}}{\lambda_{1}^{n k}} \Rightarrow \sum_{i=1}^{N} \alpha_{i} \frac{\lambda_{i}^{n k}}{\lambda_{1}^{n k}}
$$

Its components are divided into four categories, namely: center subsystem, roadside subsystem, vehicle subsystem, Walker subsystems; each type also includes varying amounts of a subsystem, UNIA for identified a total of 19 subsystems; each subsystem is further decomposition equipment package. Equipment package is one of the smallest units that can be purchased in a physical structure, each of which corresponds to one or more of the logical structures ".

Intelligent transportation system in the electronic bus stop board release system platform for service object is mainly bus passengers and transit center management personnel, they can complete the information in the system to add, delete, publish, vehicle management, line, stop and the situation of the destination [8]. View display results, and other related operations, is the use of the main body of the system.

Intelligent public transportation system can be specific description: using global positioning system (GPS) for data collection, combined with transit survey, based on geographic information system (GIS) as the operation platform, automatic dispatching and command of the public transport vehicles to ensure the punctuality of the operation of the vehicle, and resorted to walker can reach the moment through the understanding of the electronic bus stop board vehicle, thereby saving travel time of passengers.

Intelligent traffic signal lamp system with fixed time model, time setting mode, time sensing mode, on-site remote control mode, remote control mode signal control mode, date and time setting, set time, induction parameter setting, cycle time, phase difference and the green signal ratio parameter setting; can self checking system, the green conflict detection, light fault detection, line fault detection; has strong input / output function can be realized in the intersection of different phases of the lamp control output and detection function [9]. In addition, the system provides a friendly man-machine interface, the user can manually switch, keyboard or remote controller to set and control the signal, as is shown by equation(4).

$$
\left\{\begin{array}{l}
\left\{x_{1}, \cdots, x_{d}\right\}=\arg \max J(x) \\
x_{i}^{T} x_{j}=0, i \neq j, i, j=1, \cdots, d
\end{array}\right.
$$

Signal control system of traffic signal control machine and the host computer should use advanced standard data communication protocol, in order to facilitate the system in the future. Signal control 
system should have the following control functions: flashing yellow, secondary pedestrian crossing control, real-time adaptive optimization control, coordinated control induction type line, multi period timing control, countdown real-time communication function, bus priority control, emergency vehicle priority control, force control, service control plans and other functions.

With the vigorous development of China's economic construction, urban population and motor vehicle ownership in the rapid growth, increasing traffic flow, traffic congestion is becoming more and more serious, traffic accidents have occurred. As the basic component of intelligent transportation system, vehicle detector plays an important role in the intelligent transportation system. At this stage, the vehicle detector has many advantages and disadvantages, such as infrared detector, magnetic detector, mechanical piezoelectric detector, magnetic frequency detector, frequency detector, video detector, etc.. Moreover, the current domestic use of the traffic lights is fixed traffic light time, and automatic switching. The red light and green light time is according to the time, crossing the east-west and North-South traffic, determined by using statistical method. Traffic police continue to observe the intersection of the two directions, according to the vehicle density and flow rate to decide whether to switch traffic lights, in order to ensure the best road traffic control status.

\section{Design and Construction of Intelligent Traffic Control Management System Based on Internet of Things RFID Technology}

The Internet of things is the radio frequency identification (RFID), infrared sensors, global positioning system (GPS, laser scanners and other information sensing equipment, according to the agreement, the any objects connected to the Internet, the exchange of information and communication, so as to realize the intelligent of object identification, location, tracking and monitoring and management of a network.

Intelligent traffic management mainly includes the traffic control equipment, such as traffic signals, ramp traffic control and dynamic traffic information card on the road (to provide real-time traffic flow and highway status information). At the same time a city or a province traffic management center needs to get the traffic flow of the whole area in order to detect accidents, dangerous weather events or other factors that have potential threat to the traffic lane [10]. To achieve this goal, the management center needs to use information technology integrated sensors, roadside equipment, vehicle detectors, cameras, information signs and other equipment to integrate information collected by the analysis.

The system takes the FPGA as the core, which is composed of a collection, processing, and automatic control for a closed loop control system. The system hardware circuit is composed of the vehicle flow detection circuit, the single chip microcomputer, the violation detection circuit, the status light, the LED display, the key, the buzzer.

PROTEUS embedded system simulation software has been noted in the design and the integration of various compiler procedures, such as it can be used in combination with Keil, Wave8000 and other compiler simulation software. Because Wave8000 is easy to use, it is with powerful software simulation and hardware simulation function, as shown in Fig. 1.

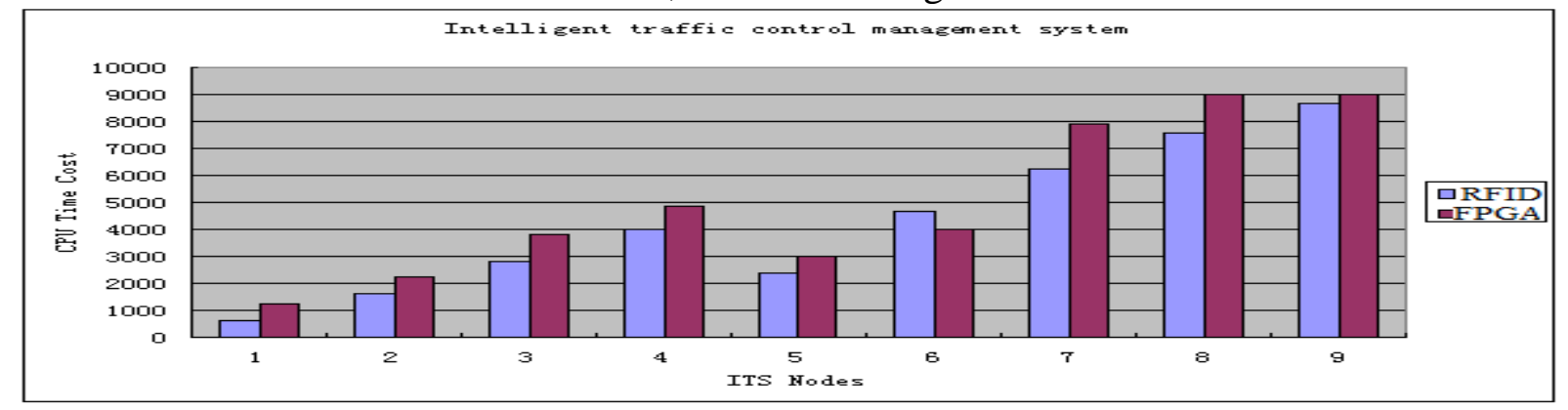

Figure 1. Comparison of intelligent traffic control management system based on Internet of things RFID with FPGA

The experiments compare the RFID technology based on Internet of things and the FPGA technology to build the intelligent traffic control management system, and the advantages and 
disadvantages of the complexity of space and time. Experiment Fig. 1 shows that the RFID in the design of intelligent traffic control signal lights CPU time consuming than the FPGA has advantages. Intelligent traffic signal lamp system is not only an independent system, but also a part of the whole area intelligent signal lamp system. Through information sharing, we can realize the linkage control of traffic signals, and make effective traffic prediction and guidance.

\section{Summary}

Traffic lights at the intersection of many cities to implement timing control, light time is set in advance good, in terms of time and space the strain of poor performance, extent caused the waste of transport resources, increased road traffic pressure. In this paper, we put forward the RFID technology based on the Internet of things to build and the actual intelligent traffic control management system. Intelligent transportation system to the human, vehicle, road and environment, such as the integration of all aspects of the transport system, so that the car, the operation of the integration and intelligent road. Intelligent transportation system is the only way to solve the traffic problems; safe, efficient, environmental protection, and low energy consumption, fast, comfortable green traffic is the development direction of the intelligent traffic.

\section{Acknowledgements}

This paper is supported by Guangdong province colleges and universities outstanding young teacher training project (Yq2013172).

\section{References}

[1] Thirumalai T., Kashwan K. R., "Split Ring Patch Antenna for FPGA Configurable RFID Applications", JNIT, Vol. 6, No. 3, pp. 47 58, 2015

[2] He Jialiang, Ouyang Dantong, Wang Haiyan, "A RFID Authentication Protocol with Index Inner Table Used in Reader", JDCTA, Vol. 6, No. 2, pp. $119 \sim$ 127, 2012.

[3] Lvqing Yang, Caili Wang, Wenhua Zeng, "Research of Matrix-based Grouping Method on Anti-collision Algorithm for RFID Tag Identification", AISS, Vol. 5, No. 13, pp. 126 133, 2013.

[4] Ming-Huo Chen, Chao-Lung Ting, Ray-I Chang, "Safe Driving Assistance by Lane-Change Detecting and Tracking for Intelligent Transportation System", IJIPM, Vol. 4, No. 7, pp. 31 38, 2013.

[5] Ren Weide, Zhang Junhu, Wang Jianping, "Design of a RFID Location Algorithm based on LANDMARC", IJACT, Vol. 5, No. 9, pp. 577 583, 2013.

[6] Hao Wang, "Wireless Sensor Networks for an Extended City Intelligent Transportation System", IJACT, Vol. 3, No. 5, pp. $300 \sim$ 307, 2011.

[7] Feng Feng, Xu Qi, "Locating Method in the Warehouse Based on Active RFID Tags", JDCTA, Vol. 7, No. 1, pp. $223 \sim 231,2013$.

[8] Zhang Duzhong, Liu Quan, Xu Wenjun, "Access Convergence of Mechanical Equipments to Heterogeneous Networking Environment in Internet of Things for Manufacturing", JCIT, Vol. 8, No. 4, pp. $902 \sim 911,2013$.

[9] Jiao Yabing, "Construction and Application of Logistics Information Tracking System Based on RFID Technology", JCIT, Vol. 8, No. 10, pp. 837 845, 2013.

[10] Zhou Libo, Zhou Minghui, Becky, "Ecological Environment Monitoring Model Based on the Internet of Things Sensing", IJACT, Vol. 5, No. 7, pp. $1152 \sim 1159,2013$. 